\title{
Steffen Höhne, Ludger Udolph (dir.), Franz Spina (1868-1938). Ein Prager Slawist zwischen Universität und politischer Öffentlichkeit
}

Köln, Weimar, Wien : Böhlau, (Intellektuelles Prag im 19. und 20. Jahrhundert, Band 2), 2012, 331 p., 44,90€

Hélène Leclerc

\section{OpenEdition}

\section{Journals}

Édition électronique

URL : http://journals.openedition.org/ifha/7535

DOI : $10.4000 /$ ifha. 7535

ISSN : 2198-8943

Éditeur

IFRA - Institut franco-allemand (sciences historiques et sociales)

Référence électronique

Hélène Leclerc, « Steffen Höhne, Ludger Udolph (dir.), Franz Spina (1868-1938). Ein Prager Slawist zwischen Universität und politischer Öffentlichkeit », Revue de l'IFHA [En ligne], Date de recension, mis en ligne le 13 décembre 2013, consulté le 22 septembre 2020. URL : http://journals.openedition.org/ifha/ 7535 ; DOl : https://doi.org/10.4000/ifha.7535

Ce document a été généré automatiquement le 22 septembre 2020.

(CIFHA 


\section{Steffen Höhne, Ludger Udolph (dir.), Franz Spina (1868-1938). Ein Prager Slawist zwischen Universität und politischer Öffentlichkeit}

Köln, Weimar, Wien : Böhlau, (Intellektuelles Prag im 19. und 20. Jahrhundert, Band 2), 2012, 331 p., 44,90€

Hélène Leclerc

Après un volume consacré au germaniste pragois August Sauer (1855-1926), le deuxième volume de la collection "Intellektuelles Prag im 19. und 20. Jahrhundert " des éditions Böhlau rend hommage à un autre acteur important de la vie intellectuelle pragoise du début du XX $\mathrm{XX}^{\mathrm{e}}$ siècle, qui fut d'ailleurs un élève d'August Sauer : le linguiste, slaviste, publiciste et homme politique allemand de Bohême Franz Spina. L'ouvrage, comportant 316 pages de texte assorties d'un index des noms de personnes et des noms de lieux, est le fruit des travaux d'un colloque organisé à Prague du 17 au 19 février 2010 au Forum culturel autrichien (Österreichisches Kulturforum). Bien que composé de quinze contributions distinctes, le livre n'en parvient pas moins à retracer une biographie d'une remarquable cohésion. Sont ainsi éclairées la carrière, la personnalité et l'œuvre de Franz Spina (activité scientifique, éditoriale, universitaire et pédagogique, politique), où il apparaît, et ce de manière frappante, que celles-ci se sont caractérisées par une dimension médiatrice et conciliatrice de tout premier ordre et par le terme-clef de « symbiose ». Aussi l'ouvrage aurait-il tout aussi bien pu s'intituler « Franz Spina, un slaviste pragois entre Tchèques et Allemands ».

Né en 1868 sujet de l'empire austro-hongrois à Trnávka (MarktTürnau) en Moravie, une commune "utraquiste", c'est-à-dire bilingue, comme le rappelle Václav Petrbok (p. 99), Franz Spina entame en 1888 des études de germanistique, philologie classique, philosophie et slavistique à l'Université allemande de Prague, où il fait sa thèse sous la direction d'August Sauer. Après divers postes d'enseignant, il obtient en 1906 une place de lecteur en langue tchèque à l'Université allemande de Prague et s'habilite en 1909 en 
langue et littérature tchèques. En 1921, il obtient un poste de professeur titulaire chargé de la langue et littérature tchèques ainsi que des autres langues et littératures slaves occidentales. En 1930, il fonde avec Gerhard Gesemann la Société allemande de recherche en slavistique à Prague (Deutsche Gesellschaft für Slavistische Forschung in Prag), qui éditera les revues Germanoslavica(1931-1937) et Slavische Rundschau (1929-1940). Après 1918 et la proclamation de la République tchécoslovaque, il s'engage véritablement en politique. Spina meurt le 17 septembre 1938, tout juste avant la conclusion des accords de Munich.

Ces éléments biographiques, rappelés par Steffen Höhne (p.10-11), laissent déjà deviner la dimension médiatrice de la personnalité et de la carrière de Franz Spina. C'est bien sous le signe de la médiation que se place en effet l'article de Steffen Höhne, qui souligne la proximité de la pensée de Spina avec des « modèles de bilinguisme » tels que celui de Bernard Bolzano (1784-1848) ou de "polyglottisme" tels que celui de J.E. Purkyně (1787-1869) (p.17), resituant le slaviste dans une "tradition bohémiste » (p. 20), puisque celui-ci «plaide [selon ses propres termes] pour 'le rapprochement culturel de deux nations culturelles'« (p.20). Steffen Höhne considère que Spina, devenu après la création de la Tchécoslovaquie en 1918 "l'homme politique du compromis", a joué un rôle de "correcteur" dans le contexte d'un discours nationaliste de plus en plus radical (p. 20). Spina fut l'un des représentants majeurs de l' "activisme " allemand, par lequel on entend la participation des Allemands au gouvernement de la Tchécoslovaquie. C'est cet aspect qu'éclaire plus particulièrement l'article d'Eduard Kubů et Jiř́ Šouša (p.141-168), qui étudient les liens étroits entretenus par Spina avec le parti agrarien allemand (Bund der Landwirte), dont il fut le président ; Spina avait notamment épousé la fille illégitime du fondateur de ce parti, au sein duquel il se fit le porte-voix des intérêts nationaux allemands avant d'évoluer en 1925 vers l'activisme et d'entrer en octobre 1926 au gouvernement. Christoph Boyer examine quant à lui la position de Spina vis-à-vis de l'activisme économique, c'est-àdire la question de la coopération des acteurs économiques allemands avec l'économie tchèque et observe que, dans ce domaine aussi, Spina se posa en avocat d'une coopération dépassant les critères d'appartenance nationale (p. 127-139). Harald Lönnecker (p. 169-212), qui rappelle que Spina fut membre de l'association étudiante pragoise Constantia, à laquelle il demeura fidèle même lorsqu'elle entama un processus de radicalisation nationale, souligne cependant que Spina prit ses distances après la Première Guerre mondiale, privilégiant alors la recherche d'un compromis entre Tchèques et Allemands.

Spina semble ainsi être entré dans l'histoire comme l'homme politique de la " symbiose " germano-tchèque. L'ensemble des contributeurs du volume fait allusion à cet emploi du terme de "symbiose» par Spina. À titre d'exemple, Karl Braun, qui explicite la réaction de Spina aux 14 points de Wilson (p. 67-80), souligne combien la frontière linguistique était loin de constituer une frontière culturelle aux yeux d'un Spina, qui misait au contraire sur la symbiose germano-tchèque (p. 74) et aurait été, en 1921 encore, "hypnotisé » par cette idée (p. 75). Michael Havlin, procédant à une intéressante généalogie du terme de symbiose, révèle néanmoins que Spina n'y aurait eu recours que trois fois entre 1917 et 1938 (p. 263); l'emploi du terme dans une interview accordée au journal français Le Matin en décembre 1926 eut un impact considérable et fit dorénavant de Spina l'apôtre de la symbiose germano-tchèque. Naturellement, la défense de la symbiose avait ses détracteurs et cette position a pu 
conduire à ce que Spina soit considéré comme un traître, comme le montre KlaasHinrich Ehlers (p. 81-96).

L'activité scientifique et pédagogique de Spina est également le sujet de plusieurs contributions. Au confluent des engagements politique et pédagogique se trouve l'engagement de Spina, avant la Première Guerre mondiale, en faveur d'une politique scolaire permettant de concilier les intérêts des Tchèques et des Allemands. Tel est le sujet de l'article de Václav Petrbok (p. 97-125), qui montre le rôle dévolu à la langue comme instrument de conciliation (p.116). Les œuvres de Spina sont analysées et leur contribution à la recherche mise en avant. Ludger Udolph (p. 39-66) retrace la carrière de Spina en tant que bohémiste et son action en vue de la création d'un lectorat de tchèque à l'Université allemande de Prague, puis d'une chaire de langue et littérature tchèques. Examinant ses travaux de bohémiste, Ludger Udolph montre combien Spina "voyait Allemands et Tchèques, leur littérature et leur culture, dans une étroite relation » (p. 46), soulignant, voire surestimant peut-être l'influence de la littérature allemande sur la littérature tchèque (p.58), mais considérant les Tchèques comme faisant partie intégrante de la civilisation ouest-européenne de par leur appartenance au christianisme romain mais aussi de part ces « influences allemandes » auxquelles ils avaient été et demeuraient exposés (p. 47). L'intérêt de Spina pour les influences allemandes sur la littérature tchèque est de nouveau souligné par Alice Stašková (p. 230). Andrea Scheller (p. 213-226) s'intéresse pour sa part à la thèse d'habilitation de Spina, consacrée à un monument de la littérature tchèque ancienne, Frantova Práva, un livre populaire écrit au début du XVI e siècle, que l'on peut comparer à Till Eulenspiegel ; ce travail universitaire, qui, de l'avis d'A. Scheller n'a nullement perdu de son actualité, constitue une contribution aux relations littéraires germano-slaves, Spina affirmant lui-même avoir voulu montrer "qu'il y a de la place pour des études consacrées aux relations germano-tchèques » (p. 224). Deux articles abordent enfin l'activité éditoriale de Spina, qui édita à Prague avec Gustav Gesemann la Slavische Rundschau (1929-1940). Dans le premier de ces deux articles, Zaur Gasimov (p. 241-259) met en perspective la Slavische Rundschau et le Wschód de Varsovie, soulignant le rôle de pont joué par la revue pragoise, qui visait à familiariser les intellectuels tchèques et slovaques avec la culture allemande et à mettre en relief les interactions étroites entre traditions culturelles germanophones et tchèques (p. 245). Cette revue devint un forum important pour de célèbres linguistes, en particulier les linguistes russes, tel Roman Jakobson, dont l'hommage prononcé lors de la mort de Spina est retranscrit à la fin du volume (p. 315-316). Spina recherchait en effet la conciliation entre Allemands et Tchèques, mais aussi entre Allemands et Slaves. L'article de Helmut Wilhelm Schaller (p. 281-296) s'intéresse plus précisément à la relation entre les deux slavistes pragois que furent Spina et Gerhard Gesemann.

Ce volume, d'une grande qualité scientifique, intéressera tout autant germanistes, bohémistes qu'historiens spécialistes de l'Europe centrale et des relations germanoslaves. 
INDEX

Index chronologique : Neuere und Neueste Geschichte

Thèmes : Kulturgeschichte

\section{AUTEUR}

HÉLÈNE LECLERC

Université Toulouse II - Le Mirail 